\title{
Study on start-up characteristics of $H$-Darrieus vertical axis wind turbines comprising NACA 4-digit series blade airfoils
}

\begin{abstract}
Installation of H-type vertical axis wind turbines is in many cases limited by the inherent start-up issues associated with this type of turbine. This could be crucial in environments with low wind speed. The aim of this study is to provide an appropriate CFD modeling setup for investigation of the start-up behavior associated with this class of turbines. For this purpose, a series of transient CFD simulations were carried out using ANSYS Fluent. In contrast with the conventional approach, whereby a constant angular velocity is specified for the rotor, in the present work, the turbine was left free to accelerate based on the torque experienced over time. Careful considerations were made regarding turbulence modeling and grid generation, which are key to ensuring accuracy in this investigation. The result of this simulation, in the form of an accelerating time series, demonstrates good agreement with the published experimental data, and the method yields a high level of accuracy, proving its usefulness for similar problems. In another attempt, the validated CFD setup was utilized to evaluate the effects of several geometric attributes of the turbine rotor on the starting characteristics. Symmetric and cambered airfoils of different thicknesses with a wide range of pitch angles were examined. The optimum start-up characteristics were observed with the use of a medium-thickness cambered airfoil, NACA2418, put to use with an outward pitch angle of $1.5^{\circ}$; this configuration decreased the start-up time while retaining the turbine's peak performance.
\end{abstract}

Keyword: Vertical axis wind turbine; VAWT; Darrieus turbine; Start-up; Self-starting; Transient CFD 